\title{
MINIMAL HYPERSURFACES OF $\mathbf{R}^{2 m}$ \\ INVARIANT BY $\mathrm{SO}(m) \times \mathrm{SO}(m)$
}

\author{
HILÁRIO ALENCAR
}

\begin{abstract}
Let $G=\mathrm{SO}(m) \times \mathrm{SO}(m)$ act in the standard way on $\mathbf{R}^{m} \times \mathbf{R}^{m}$. We describe all complete minimal hypersurfaces of $\mathbf{R}^{m} \backslash\{0\}$ which are invariant under $G$ for $m=2,3$. We also show that the unique minimal hypersurface of $\mathbf{R}^{2 m}$ which is invariant under $G$ and passes through the origin of $\mathbf{R}^{2 m}$ is the minimal quadratic cone.
\end{abstract}

\section{INTRODUCTION}

Let $G=\mathrm{SO}(m) \times \mathrm{SO}(m)$ act in the standard way on $\mathbf{R}^{2 m} \times \mathbf{R}^{2 m}$. The orbit of this action which passes through the point $(X, Y) \in \mathbf{R}^{m} \times \mathbf{R}^{m}$ is given by $S^{m-1}(|X|) \times S^{m-1}(|Y|)$, and the orbit space $\mathbf{R}^{2 m} / G$ can be represented by

$$
\pi\left(\mathbf{R}^{2 m}\right)=\left\{(x, y) \in \mathbf{R}^{2} ; x \geq 0, y \geq 0\right\},
$$

where $\pi: \mathbf{R}^{m} \times \mathbf{R}^{m} \rightarrow \mathbf{R}^{2}$ is defined by $\pi(X, Y)=(|X|,|Y|)$.

Let $M^{2 m-1}$ be a minimal hypersurface in $\mathbf{R}^{2 m}$ which is invariant under $G$. Using the methods of equivariant geometry (see [2]) we will describe the above hypersurface by studying its generating curve in $\pi\left(\mathbf{R}^{2 m}\right)$.

The generating curve of $M^{2 m-1}$ has one of the following types:

(a) the generating curve intersects perpendicularly one of the semi-axes of the orbit space;

(b) the generating curve does not intersect the boundary of the orbit space;

(c) the generating curve passes through the origin of the orbit space.

In case (c), the corresponding hypersurface passes through the origin of $\mathbf{R}^{2 m}$ and has a unique singularity at that point. This case can be completely characterized by the following result, which we will prove in $\S 4$ (see Theorem 4.1).

Let $M^{2 m-1}$ be a minimal hypersurface of $\mathbf{R}^{2 m}$ which is invariant under $G$ and passes through the origin of $\mathbf{R}^{2 m}$. Then $M^{2 m-1}$ is the minimal quadratic cone

$$
C=\left\{(X, Y) \in \mathbf{R}^{m} \times \mathbf{R}^{m} ;|X|^{2}=|Y|^{2}\right\} .
$$

This generalizes, for arbitrary $m$, a result of Barbosa-do Carmo (see [3]) for $m=2$.

Received by the editors May 12, 1989 and, in revised form, January 8, 1991.

1980 Mathematics Subject Classification (1985 Revision). Primary 53C40; Secondary 53C42.

Key words and phrases. Minimal hypersurface, orbit space, invariant hypersurface, minimal quadratic cone.

The author was partially supported by CNPq, at IMPA, Brazil. 


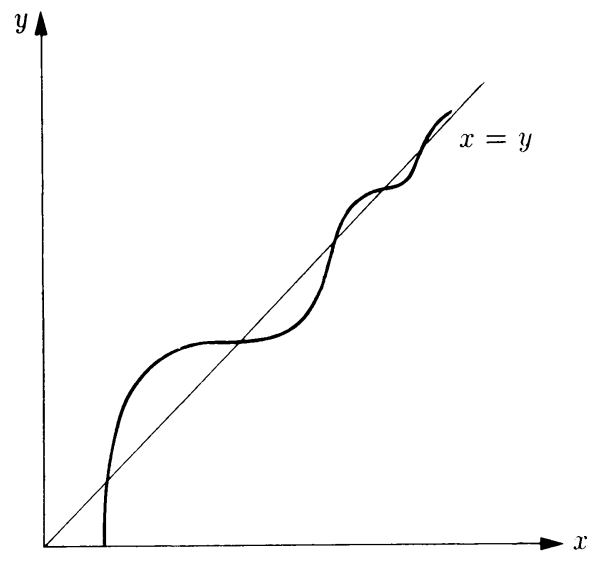

FIGURE (a)

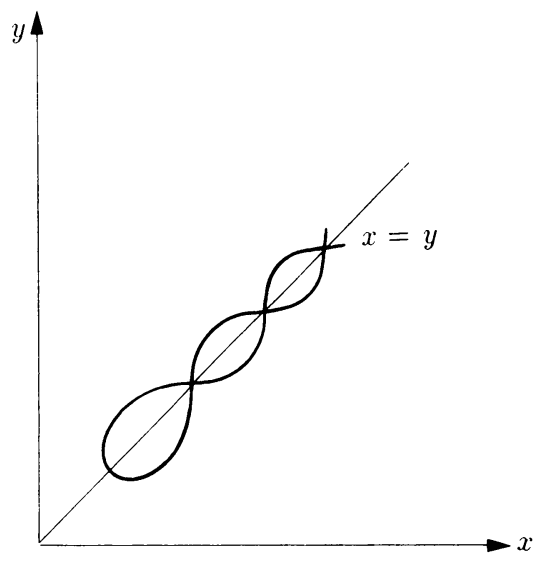

FigURE (b)

For cases (a) and (b), the hypersurfaces are regular. In the cases $m=2,3$, we have been able to obtain a rather complete description of the generating curves which are sketched in Figures (a) and (b), corresponding to cases (a) and (b), respectively (we will return to the case $m \geq 4$ at the end of this Introduction). This leads to the main result of this paper.

(1.1) Theorem. Let $M^{2 m-1}, m=2,3$, be a complete minimal hypersurface in $\mathbf{R}^{2 m} \backslash\{0\}$ which is invariant under $G$. Then:

(a) either $M^{2 m-1}$ is embedded and has the topological type of $\mathbf{R}^{m} \times S^{m-1}$, or

(b) $M^{2 m-1}$ intersects itself infinitely often (i.e., the intersection set has infinitely many connected components) and has the topological type of $\mathbf{R} \times S^{m-1} \times S^{m-1}$.

Furthermore, in cases (a) and (b), the hypersurfaces intersect the cone Coutside any compact set and it is arbitrarily close to $C$.

The paper is organized as follows. In $\S 2$, we introduce some results about the relationship between the minimal hypersurfaces of $\mathbf{R}^{2 m}$ which are invariant under $G$ and its generating curves in $\pi\left(\mathbf{R}^{2 m}\right)$. These curves satisfy the following second-order differential equation (see [8, Proposition 1]):

$$
\begin{aligned}
& x^{\prime}(s) y^{\prime \prime}(s)-x^{\prime \prime}(s) y^{\prime}(s) \\
& \quad=(m-1)\left[\left(x^{\prime}(s)\right)^{2}+\left(y^{\prime}(s)\right)^{2}\right]\left(\frac{x^{\prime}(s)}{y(s)}-\frac{y^{\prime}(s)}{x(s)}\right) .
\end{aligned}
$$

We will use an idea of Bombieri, De Giorgi, and Giusti (see [4]) and introduce parameters (see (2.3)) in the above equation which are invariant by homotheties. This transforms (1.2) in a vector field in the plane $\sigma \psi$ (see (2.7)). We then characterize the singularities of this vector field and we relate the trajectories of the field to the solutions of equation (1.2).

In $\S 3$, by using the Stable and Unstable Theorem and the existence of a Liapounov function, we obtain a complete description of the trajectories of the above vector field for $m=2,3$.

We prove that the trajectories of this field that start at saddle points and end at focus points are related to the solution curves of type (a). By using the fact 
that such curves satisfy the differential equation (1.2), we prove that they have the properties sketched in Figure (a).

Similarly, the trajectories of the vector field that start and end at focus points are related to the solution curves of type (b). In this case, we first look at the intersection points of the trajectories of the vector field with the lines $\sigma+\psi=$ $2 k \pi$ and $\psi-\sigma=(2 j+1) \pi, k$ and $j$ integers. We show that such intersection points correspond to the maximum and minimum of the angle that the position vector of the generating curve makes with $x$-axis (see Proposition 3.3); through a careful analysis (see Lemma 3.6), we then obtain that the curves of type (b) are self-intersecting curves.

Finally in $\S 4$, we prove Theorem 4.1 . The idea of the proof is to use the power series of the solution of equation (1.2). By using induction on the coefficients of the series, we prove that the unique solution which passes through the origin is the bisector of the orbit space.

For $m \geq 4$, the vector field exhibits other types of singularities and our method for the analysis of the trajectories does not seem to apply. However, by using techniques similar to those of the proof of part (a) of Theorem 1.1, we have proved in [1] (see Theorem 5.1) that:

For $m \geq 4$, the complete minimal hypersurfaces of $\mathbf{R}^{2 m}-\{0\}$, which are invariant by $\mathrm{SO}(m) \times \mathrm{SO}(m)$ and have the topological type of $\mathbf{R}^{m} \times S^{m-1}$, are embedded and foliate $\mathbf{R}^{2 m} \backslash C$. In particular, such hypersurfaces are stable.

This is part of my Doctoral Dissertation at IMPA. I want to thank M. do Carmo for his orientation. Thanks also are due to G. Thorbergsson, M. Alves, and P. Carrião for conversations.

\section{Preliminaries}

The solution curves of (1.2) are invariant by homotheties. Therefore, since minimal hypersurfaces are again minimal after homotheties, we can follow the method developed in [4] for the study of the equation (1.2). Observe that if a curve $(x, y)$ is a solution of $(1.2)$, then the curve $(y, x)$ is also a solution.

For completeness, we will now present this method.

We can suppose without loss of generality that the solutions of (1.2) are parametrized by arc length. Hence, equation (1.2) can be written as

$$
x^{\prime}(s) y^{\prime \prime}(s)-x^{\prime \prime}(s) y^{\prime}(s)=(m-1)\left(\frac{x^{\prime}(s)}{y(s)}-\frac{y^{\prime}(s)}{x(s)}\right) .
$$

Let $\eta$ be a solution curve of (2.1). Define functions $\theta(s)$ and $\varphi(s)$ by

$$
\begin{aligned}
\cos \varphi(s)=\frac{x(s)}{\sqrt{[x(s)]^{2}+[y(s)]^{2}}}, & \sin \varphi(s)=\frac{y(s)}{\sqrt{[x(s)]^{2}+[y(s)]^{2}}}, \\
\cos \theta(s)=x^{\prime}(s), & \sin \theta(s)=y^{\prime}(s),
\end{aligned}
$$

and introduce the parameters:

$$
\varphi(s)=\arctan \frac{y(s)}{x(s)}, \quad \theta(s)=\arctan \frac{y^{\prime}(s)}{x^{\prime}(s)},
$$

which are invariant by the homotheties:

$$
x(s) \mapsto \lambda x(s), \quad y(s) \mapsto \lambda y(s), \quad \lambda>0
$$


By using (2.3) and (2.2) we obtain

$$
\varphi^{\prime}=\frac{1}{\sqrt{x^{2}+y^{2}}} \sin (\theta-\varphi) .
$$

Since $\eta$ satisfies (2.1), we obtain from (2.3) and (2.2) that

$$
\theta^{\prime}=2 \frac{m-1}{\sqrt{x^{2}+y^{2}}} \frac{\cos (\theta+\varphi)}{\sin (2 \varphi)} \text {. }
$$

Hence, by the above expression and (2.4), we have the following relation:

$$
-2(m-1) \varphi^{\prime} \cos (\varphi+\theta)+\theta^{\prime} \sin (2 \varphi) \sin (\theta-\varphi)=0 \text {. }
$$

Setting

$$
\sigma=\theta-3 \varphi+\frac{\pi}{2}, \quad \psi=\theta+\varphi-\frac{\pi}{2}
$$

we obtain

$$
\varphi=\frac{\psi-\sigma+\pi}{4}, \quad \theta=\frac{3 \psi+\sigma+\pi}{4} .
$$

Therefore,

$$
\begin{gathered}
-2(m-1) \varphi^{\prime} \cos (\varphi+\theta)=\frac{1}{2}(m-1)\left(\psi^{\prime}-\sigma^{\prime}\right) \sin \psi, \\
\theta^{\prime} \sin 2 \varphi \sin (\theta-\varphi)=\frac{1}{8}\left(3 \psi^{\prime}+\sigma\right)(\sin \psi+\sin \sigma) .
\end{gathered}
$$

Thus, by using (2.5), we obtain the following vector field in the plane in the coordinates $(\sigma, \psi)$ :

$$
\begin{aligned}
& \frac{d \sigma}{d s}=-\frac{3}{2} \sin \sigma-\left(2(m-1)+\frac{3}{2}\right) \sin \psi, \\
& \frac{d \psi}{d s}=\frac{1}{2} \sin \sigma-\left(2(m-1)-\frac{1}{2}\right) \sin \psi .
\end{aligned}
$$

Through the study of the vector field (2.7) we will describe some properties of the differential equation (2.1).

We will first characterize the singular points of the above vector field. The terminology we use here can be found in [6].

The singular points of (2.7) are of the form $(\sigma, \psi)=(j \pi, k \pi), j, k \in \mathbf{Z}$. Since the sine function is periodic, it suffices to study the following singular points: $(0,0),(\pi, \pi),(-\pi,-\pi),(-\pi, \pi)$, and $(0,-\pi)$.

By writing the vector field (2.7) as

$$
X(\sigma, \psi)=\left(-\frac{3}{2} \sin \sigma-\left(2(m-1)+\frac{3}{2}\right) \sin \psi, \frac{1}{2} \sin \sigma-\left(2(m-1)-\frac{1}{2}\right) \sin \psi\right)
$$

and by denoting by $D X(p)$ the differential of the mapping $X$ at $p$, we obtain that the eigenvalues of the matrix $D X(0,0)$ are:

$$
\lambda_{1}(0,0)=\frac{1-2 m+\sqrt{\Delta}}{2}, \quad \lambda_{2}(0,0)=\frac{1-2 m-\sqrt{\Delta}}{2},
$$

where $\Delta=4(m-1)^{2}-12(m-1)+1$.

In the same way, we obtain for the eigenvalues of $D X$ at the other singular points:

$$
\begin{gathered}
\lambda_{1}(\pi, \pi)=\lambda_{1}(-\pi,-\pi)=\lambda_{1}(-\pi, \pi)=\lambda_{1}(\pi,-\pi)=\frac{2 m-1+\sqrt{\Delta}}{2}, \\
\lambda_{2}(\pi, \pi)=\lambda_{2}(-\pi,-\pi)=\lambda_{2}(-\pi, \pi)=\lambda_{2}(\pi,-\pi)=\frac{2 m-1-\sqrt{\Delta}}{2}, \\
\lambda_{1}(\pi, 0)=\lambda_{1}(-\pi, 0)=2, \quad \lambda_{2}(\pi, 0)=\lambda_{2}(-\pi, 0)=2-2 m, \\
\lambda_{1}(0, \pi)=\lambda_{1}(0,-\pi)=2 m-2, \quad \lambda_{2}(0, \pi)=\lambda_{2}(0,-\pi)=-2 .
\end{gathered}
$$


If we consider $m$ to be a nonnegative continuous parameter, we can write:

$$
\begin{array}{ll}
\Delta>0 & \text { if } m>\frac{5}{2}+\sqrt{2} \text { or } m<\frac{5}{2}-\sqrt{2} \\
\Delta<0 & \text { if } \frac{5}{2}-\sqrt{2}<m<\frac{5}{2}+\sqrt{2} \\
\Delta=0 & \text { if } m=\frac{5}{2}-\sqrt{2} \text { or } m=\frac{5}{2}+\sqrt{2} .
\end{array}
$$

We will only be interested in the characterization of the singular points for the cases where $m$ is an integer greater than or equal to 2 .

First of all, we characterize the singular points whose eigenvalues depend on $\Delta$ with $m=2,3$. In this case, $\Delta<0$.

Thus, $\lambda_{1}(0,0)$ and $\lambda_{2}(0,0)$ are conjugate complex numbers with negative real part, that is, $(0,0)$ is a stable focus point. In addition, $\lambda_{1}(\pi, \pi)=$ $\lambda_{1}(-\pi,-\pi)=\lambda_{1}(-\pi, \pi)=\lambda_{1}(\pi,-\pi)$ and $\lambda_{2}(\pi, \pi)=\lambda_{2}(-\pi,-\pi)=\lambda_{2}(-\pi, \pi)$ $=\lambda_{2}(\pi,-\pi)$ are also conjugate complex numbers. But in this latter case the real part is positive, that is, $(\pi, \pi),(-\pi,-\pi),(-\pi, \pi)$, and $(\pi,-\pi)$ are unstable focus points.

Now we analyze the singular points whose eigenvalues do not depend on $\Delta$.

Since $m \geq 2$, we have that

$$
\begin{aligned}
& \lambda_{1}(\pi, 0)=\lambda_{1}(-\pi, 0)>0>\lambda_{2}(\pi, 0)=\lambda_{2}(-\pi, 0), \\
& \lambda_{1}(0, \pi)=\lambda_{1}(0,-\pi)>0>\lambda_{2}(0, \pi)=\lambda_{2}(0,-\pi) ;
\end{aligned}
$$

in other words, $(\pi, 0),(-\pi, 0),(0, \pi)$, and $(0,-\pi)$ are saddle points.

In order to translate the behavior of (2.7) into the behavior of equation (2.1), we need the following lemma.

(2.8) Lemma. Let $(x(s), y(s))$ be a solution of $(2.1)$ and let $(\sigma(s), \psi(s))$ be a trajectory of (2.7) in the plane $\sigma \psi$, where $\sigma(s)$ and $\psi(s)$ are given by equation (2.6). Then we have the following properties:

(i) $\sigma=\pi, \psi=0 \Rightarrow x>0, y=0, x^{\prime}>0, y^{\prime}>0$;

(ii) $\sigma=\psi \Rightarrow x=y ; \quad \sigma=-\psi \Rightarrow \varphi=\theta$;

(iii) $\sigma=\psi=0 \Rightarrow x=y>0, x^{\prime}=y^{\prime}>0$;

(iv) $\sigma=0,0<\psi<\pi \Rightarrow y>x$

(v) $\sigma=0,-\pi<\psi<0 \Rightarrow y<x$;

(vi) $0<\psi<\sigma<\pi \Rightarrow 0<\varphi<\frac{\pi}{4} \Rightarrow y<x$.

Proof. (i), (ii), and (iii) follow from (2.6) and (2.2).

In order to prove (iv), set $\sigma=0$ in (2.6) to obtain

$$
\varphi=\frac{\psi}{4}+\frac{\pi}{4} .
$$

By (2.9) and since $0<\psi<\pi$, we obtain $\tan \varphi=1+2 \varepsilon$, where $\varepsilon=$ $\tan (\psi / 4) /(1-\tan (\psi / 4))>0$. By using (2.3) we can conclude $\frac{y}{x}=1+2 \varepsilon$, that is, $y>x$. This proves (iv).

Similarly, taking into account that $-\pi<\psi<0$ implies $\varepsilon<0$, we can prove (v).

Finally, (vi) follows from (2.6) and (2.5). In fact, by using (2.6) together with the hypothesis, we obtain $0<\sigma-\psi=-4 \varphi+\pi<\pi$, that is, $0<\varphi<\frac{\pi}{4}$. So, from (2.3), $0<\frac{y}{x}<1$. This concludes the proof of the lemma. 


\section{Proof OF TheOREM 1.1}

In order to prove Theorem 1.1, we characterize the behavior of the generating curves in cases (a) and (b) of the theorem. We will need some auxiliary results.

(3.1) Lemma. Let $m=2,3$. There exists a trajectory $\gamma(s)$ in the plane $\sigma \psi$ of the vector field (2.7), with the following properties:

(i) $\gamma(-\infty)=(\pi, 0), \gamma(+\infty)=(0,0)$;

(ii) The straight lines $\sigma=0, \psi=0, \sigma=\psi$, and $\sigma=-\psi$ intersect the curve $\gamma$ infinitely often.

Proof. We analyze the behavior of the vector field in $\sigma \psi$ determined by (2.7) on the following segments: $l_{1}=\{0<\sigma<\pi ; \psi=0\}, l_{2}=\{0<\psi<\pi ; \sigma=0\}$, $l_{3}=\left\{0<\psi=-\frac{\sigma}{2}+\frac{\pi}{2}<\frac{\pi}{2}\right\}$, and $l=\{0<\psi=\sigma+\pi<\pi\}$.

On $l_{1}$, we have

$$
\frac{d \sigma}{d s}=-\frac{3}{2} \sin \sigma<0, \quad \frac{d \psi}{d s}=\frac{1}{2} \sin \sigma>0 .
$$

On $l_{2}$, we have

$$
\frac{d \sigma}{d s}=-\left(2(m-1)+\frac{3}{2}\right) \sin \psi<0, \quad \frac{d \psi}{d s}=-\left(2(m-1)-\frac{1}{2}\right) \sin \psi<0 .
$$

On $l_{3}$, we have

$$
\begin{aligned}
& \frac{d \sigma}{d s}=-\left(3 \cos \psi+\left(2(m-1)-\frac{1}{2}\right)\right) \sin \psi<0, \\
& \frac{d \psi}{d s}=\left(\cos \psi-\left(2(m-1)-\frac{1}{2}\right)\right) \sin \psi<0 .
\end{aligned}
$$

Finally on $l$, we have

$$
\frac{d \sigma}{d s}=2(m-1) \sin \sigma<0, \quad \frac{d \psi}{d s}=2(m-1) \sin \sigma<0 .
$$

Therefore, $l$ is a trajectory of the vector field (2.7).

Observe that if $(\sigma(s), \psi(s))$ is a trajectory of $(2.7)$, so is $(-\sigma(s),-\psi(s))$. Therefore, we have a sketch (see Figure 3.1) of the behavior of the vector field on the segments $l_{1}, l_{2}, l_{3}, l$ and on the segments that are symmetric to these relative to the origin: $l_{1}^{-}, l_{2}^{-}, l_{3}^{-}$, and $l^{-}$.

Now, let $E^{s}(p)$ and $E^{u}(p)$ be the eigenspaces associated to the negative and positive eigenvalues, respectively, of $D X(p)$ at the singular point $p$. Since the slope of $E^{u}(\pi, 0)$ is $-1 / 4(m-1)+3$, we have, by using the Unstable Manifold Theorem (see $[9$, p. 75]), that there is a trajectory $\gamma$ with the following properties: the initial segment of $\gamma$ is between $l_{1}$ and $l_{3}$ and $\gamma(-\infty)=(\pi, 0)$. In addition, the curve $\gamma$ is always contained in the region $R$ bounded by $l_{3},\left\{\frac{\pi}{2} \leq \psi \leq \pi ; \sigma=0\right\}, l, l_{3}^{-},\left\{-\pi \leq \psi \leq-\frac{\pi}{2} ; \sigma=0\right\}$, and $l^{-}$(see Figure $3.1)$.

We will now prove that $\gamma(+\infty)=(0,0)$. Let

$$
V(\sigma, \psi)=\frac{1}{4(m-1)+3} \sin ^{2} \frac{\sigma}{2}+\sin ^{2} \frac{\psi}{2},
$$

where $(\sigma, \psi) \in(-\pi, \pi) \times(-\pi, \pi)$. Note that $V(0,0)=0, V(\sigma, \psi)>0$ for every $(\sigma, \psi) \neq(0,0)$, and $\frac{d V}{d s}<0$ for every $(\sigma(s), \psi(s)) \neq(0,0)$, that is, $V$ 


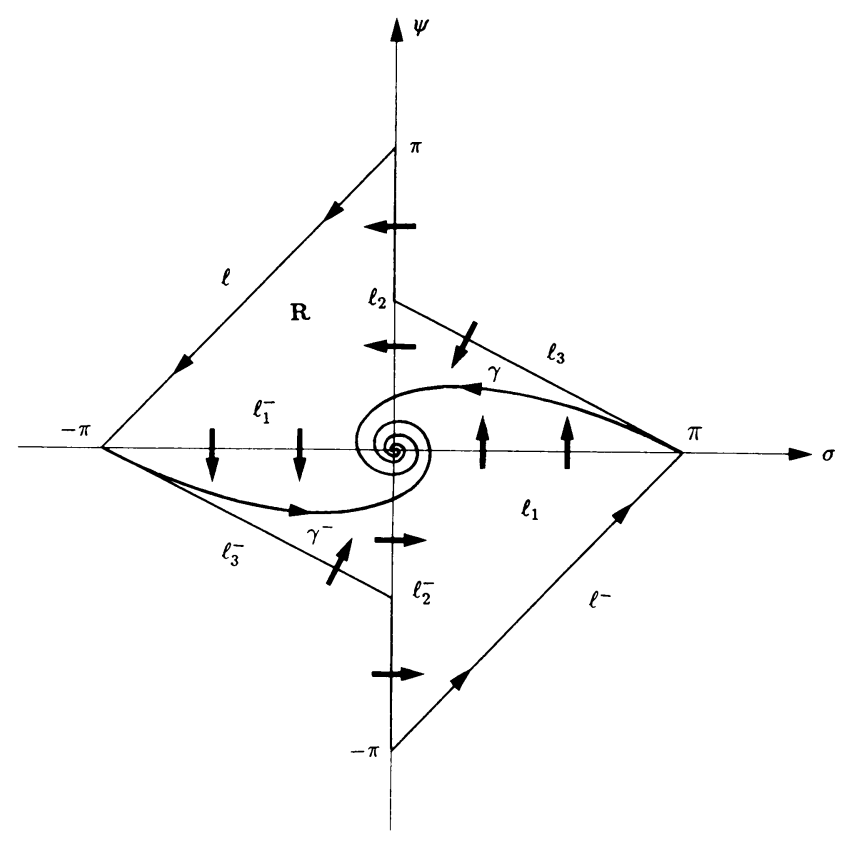

FIGURE 3.1

is a strict Liapounov function for the singularity $(0,0)$. Thus, there exists no closed orbit in $R$.

On the other hand, since the slopes of $E^{u}(0, \pi), E^{s}(-\pi, 0), E^{u}(0,-\pi)$, and $E^{s}(\pi, 0)$ are all equal to 1 , and the slopes of $E^{s}(0, \pi), E^{u}(-\pi, 0)$, $E^{s}(0,-\pi)$, and $E^{u}(\pi, 0)$ are all equal to $-1 / 4(m-1)+3$, we obtain, by using the Stable and Unstable Manifolds Theorem (see [9, p. 75]), that $l, l^{-}$, $\gamma^{-}$, and $\gamma$ are the unique trajectories contained in $R$ and in the stable or unstable manifolds of the points $(0, \pi),(-\pi, 0),(0,-\pi)$, and $(\pi, 0)$. But, by the Jordan Curve Theorem, $\gamma$ cannot accumulate in $l, l^{-}$, or $\gamma^{-}$. Then, by using the Poincaré-Bendixon Theorem (see [6, Theorem 1.2, p. 54]), we have that $\gamma(+\infty)=(0,0)$. This proves (i) of the lemma.

The proof of (ii) follows from the fact that $(0,0)$ is a focus point.

By using the above lemma, the fact that $X(\sigma+\pi, \psi+\pi)=-X(\sigma, \psi)$, the periodicity of the singular points, and the Poincare-Bendixon Theorem, we can describe completely the phase plane of the vector field (see Figure 3.2). In particular, there is a trajectory $\alpha$ in the plane $\sigma \psi$ of the vector field (2.7) such that $\alpha(-\infty)=(\pi, \pi)$ and $\alpha(+\infty)=(0,0)$.

Through the trajectories $\gamma$ (see Lemma 3.1) and $\alpha$ we will describe the behavior of the solution curves of $(2.1)$.

The trajectory $\gamma(s)$ corresponds to a family $\left\{\gamma_{\lambda}(s)\right\}, \lambda>0,-\infty<s<\infty$, of homothetic curves, parametrized by arc length in the plane $x y$, which are solutions of (2.1). Each curve belonging to the family $\left\{\gamma_{\lambda}\right\}$ has, by Lemmas 3.1 and 2.8, the initial point in $\left(x_{0}, 0\right), x_{0} \neq 0$, and is perpendicular to the axis $y=0$ at this point. Thus, by Proposition 1 in [7], the curve $\gamma_{\lambda}$ is completely determined by initial conditions at any point of the region $\{(x, y) ; x>0$, $y \geq 0\}$. 


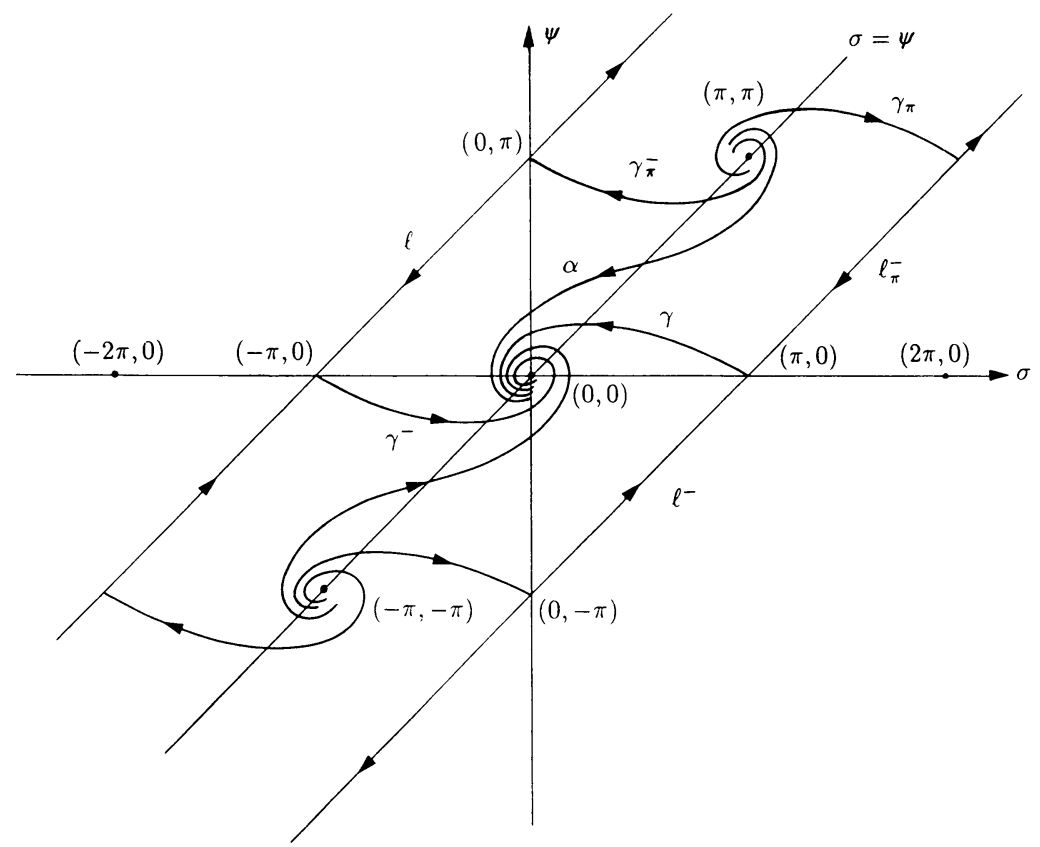

FIGURE 3.2

The trajectory $\alpha(s)$ corresponds to a family $\left\{\alpha_{\lambda}(s)\right\}, \lambda>0,-\infty<s<\infty$, of homothetic curves, parametrized by arc length in the plane $x y$, which are solutions of (2.1). By the behavior of the vector field (2.7) and by Lemma 2.8, we have that each curve $\alpha_{\lambda}$ belonging to the family $\left\{\alpha_{\lambda}\right\}$ does not intersect the boundary of $\pi\left(\mathbf{R}^{2 m}\right)$.

Given a trajectory $\beta$ of (2.7) we will denote by $\beta_{\pi}$ the trajectory of the vector field which is the translation of $\beta$ by $(\pi, \pi)$.

By using (2.6), we obtain that the trajectories $l_{\pi}^{-}$and $l$ in the plane $\sigma \psi$ correspond to the semi-axes $0 x$ and $0 y$ in the plane $x y$, respectively. In addition, $\gamma^{-}(-\infty)=(-\pi, 0)$ implies $x=0, y>0$.

On the other hand, the trajectories $\gamma_{\pi}^{-}$and $\gamma_{\pi}$ are translations of the trajectories $\gamma^{-}$and $\gamma$, respectively. Therefore, in order to describe the behavior of the solution curves of $(2.1)$ with $m=2,3$, which either do not intersect the semi-axes $0 x$ and $0 y$ or intersect perpendicularly the positive semi-axis $0 x$, it suffices to describe the behavior of the curves $\alpha_{\lambda}$ and $\gamma_{\lambda}$, respectively.

We begin analyzing the behavior of the curve $\gamma_{\lambda}$ through the following lemma.

(3.2) Lemma. The curve $\gamma_{\lambda}(s)$ has the following properties:

(i) $\gamma_{\lambda}$ is embedded;

(ii) $\gamma_{\lambda}$ intersects the diagonal $x=y$ infinitely often and is asymptotic to this diagonal.

Proof. (i) The curve $\gamma_{\lambda}(s)=(x(s), y(s))$ remains completely determined by the following initial conditions: $x(-\infty)=x_{0} \neq 0, y(-\infty)=0, x^{\prime}(-\infty)=0$, $y^{\prime}(-\infty)=1$.

Since, by Proposition 3 in [8], $I(s)=(x(s))^{m-1} y^{\prime}(s)$ is a monotonically increasing function of $s$, we have from (2.2) that $(x(s))^{m-1} \geq(x(s))^{m-1} \sin \theta(s)$ $=I(s) \geq I(-\infty)=x_{0}^{m-1}$, that is, $x(s) \geq x_{0}$. 
Similarly, we obtain $x(s) \geq x\left(s_{0}\right)$ if $s>s_{0}, x\left(s_{0}\right)=x_{0} \neq 0, y\left(s_{0}\right)=y_{0}$, $x^{\prime}\left(s_{0}\right)=0$, and $y^{\prime}\left(s_{0}\right)=1$.

By again using Proposition 3 in [8] we now know that $J(s)=(y(s))^{m-1} x^{\prime}(s)$ is a monotonically increasing function of $s$. Thus, if $x\left(s_{1}\right)=x_{1} \neq 0, y\left(s_{1}\right)=$ $y_{1} \neq 0, x^{\prime}\left(s_{1}\right)=1$, and $y^{\prime}\left(s_{1}\right)=0$, we have, using $(2.2)$, that $(y(s))^{m-1} \geq$ $(y(s))^{m-1} \cos \theta(s)=J(s) \geq J\left(s_{1}\right)=\left(y\left(s_{1}\right)\right)^{m-1}$ with $s>s_{1}$. So $y(s) \geq y\left(s_{1}\right)$.

We conclude that $\gamma_{\lambda}$ does not intersect itself, that is, $\gamma_{\lambda}$ is embedded. This proves (i).

(ii) Since the line $\sigma=\psi$ intersects the trajectory $\gamma$ infinitely often (see Lemma 3.1) we obtain, using Lemma 2.8, that the line $x=y$ intersects $\gamma_{\lambda}$ infinitely often. In addition, $\gamma(+\infty)=(0,0)$ implies (see Lemma 2.8) that $x=y$ and $x^{\prime}=y^{\prime}>0$, that is, $\gamma_{\lambda}$ is asymptotic to the diagonal $x=y$.

Before giving the characterization of the behavior of the curve $\alpha_{\lambda}$, we need the following proposition.

(3.3) Proposition. Let $\varphi$ be the angle in the plane $x y$ of the position vector of the curve $\alpha_{\lambda}$ with the $0 x$-axis (see (2.3)). Then the local maximum and minimum values of $\varphi$ are attained when $\sigma+\psi=2 k \pi$ or $\psi-\sigma=(2 j+1) \pi$, where $k, j \in \mathbf{Z}$. Furthermore,

$$
\varphi=\frac{\psi}{2}+\frac{\pi}{4}
$$

when $k=0$.

Proof. By (2.6) we obtain that

$$
\varphi=\frac{\psi-\sigma}{4}+\frac{\pi}{4}
$$

Thus $\varphi^{\prime}=0$ is equivalent to $\sigma^{\prime}=\psi^{\prime}$ and from (2.7) we obtain that $\sigma^{\prime}=\psi^{\prime}$ is equivalent to $\sin \sigma=\sin (-\psi)$. So $\sigma+\psi=2 k \pi$ or $\psi-\sigma=(2 j+1) \pi$. Now (3.4) follows from (3.5) when $k=0$. This proves the proposition.

We will now present some properties of the curve $\alpha_{\lambda}$. As we saw before, such a curve does not intersect the boundary of the orbit space.

(3.6) Lemma. The curve $\alpha_{\lambda}(s)$ has the following properties:

(i) $\alpha_{\lambda}$ intersects the diagonal $x=y$ infinitely often and is asymptotic to the diagonal when $s$ tends to $+\infty$ and to $-\infty$;

(ii) $\alpha_{\lambda}$ intersects itself.

Proof. (i) Since the trajectory $\alpha$ intersects the diagonal $\sigma=\psi$ infinitely often we obtain, by Lemma 2.8 , that $\alpha_{\lambda}$ intersects the diagonal $x=y$ infinitely often. Thus, $\alpha(-\infty)=(\pi, \pi)$ implies, by Lemma 2.8 , that $x(s)=y(s)$ and $x^{\prime}(s)=y^{\prime}(s)<0$ when $s$ tends to $-\infty$. Similarly, $\alpha(+\infty)=(0,0)$ implies that $x(s)=y(s)$ and $x^{\prime}(s)=y^{\prime}(s)>0$ when $s$ tends to $+\infty$. This proves (i).

(ii) Let $\left\{\left(\sigma\left(s_{2 m-1}^{-}\right), \psi\left(s_{2 m-1}^{-}\right)\right)\right\}_{m \geq 1}$ and $\left\{\left(\sigma\left(s_{2 m-1}^{+}\right), \psi\left(s_{2 m-1}^{+}\right)\right)\right\}_{m \geq 1}$ be sequences of points of the trajectory $\alpha$ (see Figure 3.3) such that:

$$
\begin{array}{ll}
\frac{\pi}{2}<\psi\left(s_{1}^{-}\right)<\psi\left(s_{3}^{-}\right)<\cdots<\pi, & \sigma\left(s_{2 m-1}^{-}\right)+\psi\left(s_{2 m-1}^{-}\right)=2 \pi, \\
\frac{3 \pi}{2}>\psi\left(s_{1}^{+}\right)>\psi\left(s_{3}^{+}\right)>\cdots>\pi, & \sigma\left(s_{2 m-1}^{+}\right)+\psi\left(s_{2 m-1}^{+}\right)=2 \pi,
\end{array}
$$

respectively. 


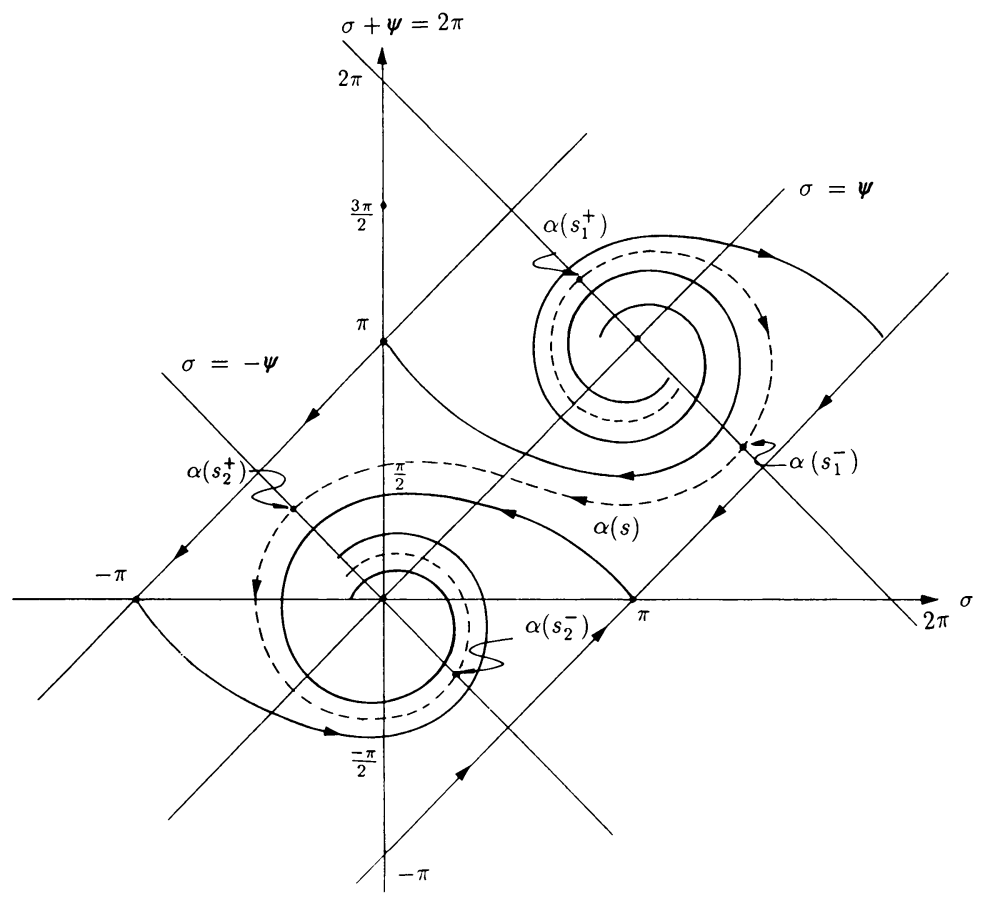

FIGURE 3.3

Let $\left\{\left(\sigma\left(s_{2 m}^{-}\right), \psi\left(s_{2 m}^{-}\right)\right)\right\}_{m \geq 1}$ and $\left\{\left(\sigma\left(s_{2 m}^{+}\right), \psi\left(s_{2 m}^{+}\right)\right)\right\}_{m \geq 1}$ be sequences of points of the trajectory $\alpha$ (see Figure 3.3) such that:

$$
\begin{array}{cc}
\frac{-\pi}{2}<\psi\left(s_{2}^{-}\right)<\psi\left(s_{4}^{-}\right)<\cdots<0, & \sigma\left(s_{2 m}^{-}\right)+\psi\left(s_{2 m}^{-}\right)=0, \\
\frac{\pi}{2}>\psi\left(s_{2}^{+}\right)>\psi\left(s_{4}^{+}\right)>\cdots>0, & \sigma\left(s_{2 m}^{+}\right)+\psi\left(s_{2 m}^{+}\right)=0,
\end{array}
$$

respectively.

If we translate the points $\alpha\left(s_{2 m-1}^{-}\right)$and $\alpha\left(s_{2 m-1}^{+}\right)$, by $(-\pi,-\pi)$, we obtain

$$
\begin{aligned}
& \psi\left(s_{1}^{-}\right)-\pi<\psi\left(s_{2}^{-}\right)<\psi\left(s_{3}^{-}\right)-\pi<\cdots<\psi\left(s_{2 m-1}^{-}\right)-\pi<\psi\left(s_{2 m}^{-}\right)<\cdots, \\
& \psi\left(s_{2}^{+}\right)>\psi\left(s_{1}^{+}\right)-\pi>\psi\left(s_{4}^{+}\right)>\cdots>\psi\left(s_{2 m}^{+}\right)>\psi\left(s_{2 m-1}^{+}\right)-\pi>\cdots .
\end{aligned}
$$

By using (3.5) we obtain that the value of $\varphi$ is invariant if we translate a point $(\sigma, \psi)$ by $(\pi, \pi)$. Thus, from (3.7), we obtain

$$
\begin{aligned}
& \varphi\left(s_{1}^{-}\right)<\varphi\left(s_{2}^{-}\right)<\cdots<\varphi\left(s_{2 m-1}^{-}\right)<\varphi\left(s_{2 m}^{-}\right)<\cdots, \\
& \varphi\left(s_{2}^{+}\right)>\varphi\left(s_{1}^{+}\right)>\cdots>\varphi\left(s_{2 m}^{+}\right)>\varphi\left(s_{2 m-1}^{+}\right)>\cdots .
\end{aligned}
$$

It follows from Proposition 3.3 that $s_{m}^{-}$and $s_{m}^{+}, m \geq 1$, are points of local minima and local maxima of $\varphi$, respectively. Furthermore, by (3.8), $s_{1}^{-}$and $s_{2}^{+}$are points of global minima and global maxima of $\varphi$, respectively.

Now we are going to show, by again using (3.8), that $\alpha_{\lambda}$ intersects itself. In fact, consider the curve segment $\alpha_{\lambda}$ that joins the points $\alpha_{\lambda}\left(s_{1}^{-}\right)$and $\alpha_{\lambda}\left(s_{2}^{+}\right)$. Then $\alpha_{\lambda}\left(s_{1}^{+}\right)$either belongs to the cone determined by $O \alpha_{\lambda}\left(s_{2}^{+}\right), \alpha_{\lambda}\left(s_{1}^{-}\right) \alpha_{\lambda}\left(s_{2}^{+}\right)$, and $O \alpha_{\lambda}\left(s_{1}^{-}\right)$or not. In the first case, since $\varphi\left(s_{1}^{-}\right)<\varphi\left(s_{2 m-1}^{-}\right), \varphi\left(s_{2 m-1}^{+}\right)<$ 
$\varphi\left(s_{2}^{+}\right), m \geq 2$, and (i) holds, we obtain that $\alpha_{\lambda}$ intersects itself. In the second case, since $\varphi\left(s_{1}^{-}\right)<\varphi\left(s_{2 m}^{-}\right), m \geq 1, \varphi\left(s_{2 m}^{+}\right)<\varphi\left(s_{1}^{+}\right), m \geq 2$, and (i) holds, we obtain that $\alpha_{\lambda}$ also intersects itself. This proves our assertion and Lemma 3.6.

Proof of Theorem 1.1. (a) Since $M^{2 m-1}$ is of the topological type of a $\mathbf{R}^{m} \times$ $S^{m-1}$, we obtain that its generating curve belongs to the family $\left\{\gamma_{\lambda}\right\}$. Therefore, by Lemma 3.2, we know that its generating curve is embedded. It follows, by using Lemma 1.4 in [5], that $M^{2 m-1}$ is embedded. This proves (a).

(b) Since $M^{2 m-1}$ is of the topological type of $\mathbf{R} \times S^{m-1} \times S^{m-1}$, its generating curve belongs to the family $\left\{\alpha_{\lambda}\right\}$. Thus, by Lemma 3.6, we know that its generating curve intersects itself. So, by using Lemma 1.4 in [5], $M^{2 m-1}$ intersects itself. This proves (b).

The fact that $M^{2 m-1}$ intersects the minimal quadratic cone outside of any compact, and it is arbitrarily close to this cone follows by Lemma 3.2(ii) and Lemma 3.6(i). This concludes the proof of Theorem 1.1

\section{The Minimal QUADRATIC CONE}

In this section we prove the following theorem.

(4.1) Theorem. Let $M^{2 m-1}, m \geq 2$, be a minimal hypersurface of $\mathbf{R}^{2 m}$ such that it is invariant under $G$ and passes through the origin of $\mathbf{R}^{2 m}$. Then $M^{2 m-1}$ is the minimal quadratic cone.

In order to prove the above theorem, we need the following result.

(4.2) Lemma. If $x(s)$ and $y(s)$ are real analytic functions satisfying (2.1) with $x(0)=y(0)=0$, then $x(s)=y(s)=\frac{\sqrt{2}}{2} s$.

Proof. We can write $x(s)=\sum_{n=1}^{\infty} a_{n} s^{n}$ and $y(s)=\sum_{n=1}^{\infty} b_{n} s^{n}$. Therefore,

$$
\begin{aligned}
& x(s) x^{\prime}(s)=\sum_{n=1}^{\infty}\left(\sum_{k=0}^{n-1}(k+1) a_{k+1} a_{n-k}\right) s^{n}, \\
& y(s) y^{\prime}(s)=\sum_{n=1}^{\infty}\left(\sum_{k=0}^{n-1}(k+1) b_{k+1} b_{n-k}\right) s^{n} .
\end{aligned}
$$

Thus,

$$
x(s) x^{\prime}(s)-y(s) y^{\prime}(s)=\left(a_{1}^{2}-b_{1}^{2}\right) s+\sum_{n=2}^{\infty} c_{n} s^{n},
$$

where

$$
c_{n}=\sum_{k=0}^{n-1}(k+1)\left(a_{k+1} a_{n-k}-b_{k+1} b_{n-k}\right), \quad n \geq 2 .
$$

Similarly,

$$
x(s) y(s)\left(x^{\prime \prime}(s) y^{\prime}(s)-x^{\prime}(s) y^{\prime \prime}(s)\right)=\sum_{n=2}^{\infty}\left(\sum_{i=0}^{n-2} d_{i} e_{n-i-2}\right) s^{n},
$$


where

$$
\begin{aligned}
& d_{i}=\sum_{k=0}^{i}(k+2)(k+1)(i-k+1)\left(a_{k+2} b_{i-k+1}-a_{i-k+1} b_{k+2}\right), \quad i \geq 0, \\
& e_{n}=\sum_{j=0}^{n} a_{n-j+1} b_{j+1}, \quad n \geq 0 .
\end{aligned}
$$

Since $x$ and $y$ satisfy (2.1), we obtain from (4.3) and (4.5) that

$$
(m-1)\left(a_{1}^{2}-b_{1}^{2}\right) s+\sum_{n=2}^{\infty}\left((m-1) c_{n}+\sum_{i=0}^{n-2} d_{i} e_{n-i-2}\right) s^{n}=0 .
$$

Now, we use the induction method on the coefficients in order to complete the proof. It is clear from (4.7) that $a_{1}=b_{1}$. Suppose that

$$
a_{l}=b_{l}, \quad l=1,2, \ldots, n-1 .
$$

Therefore,

$$
\begin{aligned}
a_{k+2} b_{i-k+1}-a_{i-k+1} b_{k+2}=0, & i=0,1, \ldots, n-2, \\
& k=0,1, \ldots, n-3 .
\end{aligned}
$$

Since, by using (4.6),

$$
\begin{aligned}
& \sum_{i=0}^{n-2} d_{i} e_{n-i-2} \\
& =\sum_{i=0}^{n-2}\left[\left(\sum_{k=0}^{i}(k+2)(k+1)(i-k+1)\left(a_{k+2} b_{i-k+1}-a_{i-k+1} b_{k+2}\right)\right)\right. \\
& \left.\cdot\left(\sum_{j=0}^{n-i-2} a_{n-i-j-1} b_{j+1}\right)\right],
\end{aligned}
$$

we obtain, from (4.9) and (4.8),

$$
\sum_{i=0}^{n-2} d_{i} e_{n-2}=n(n-1) a_{1}^{3}\left(a_{n}-b_{n}\right) \text {. }
$$

Also from (4.4) and (4.8),

$$
c_{n}=(n+1) a_{1}\left(a_{n}-b_{n}\right) .
$$

Thus, by using (4.11), (4.10), and (4.7), we obtain

$$
a_{1}\left[(m-1)(n+1)+n(n-1) a_{1}^{2}\right]\left(a_{n}-b_{n}\right)=0 .
$$

This proves that $x(s)=y(s)$. Since $\left(x^{\prime}(s)\right)^{2}+\left(y^{\prime}(s)\right)^{2}=1$, we conclude the proof of the lemma.

Proof of Theorem 4.1. Since a minimal hypersurface in $\mathbf{R}^{2 m}$ is analytic, we obtain that its generating curve is analytic. Therefore, since the hypersurface $M^{2 m-1}$ passes through the origin of $\mathbf{R}^{2 m}$, we have that the coordinate functions of its generating curve satisfy the hypothesis of Lemma 4.2. So, the generating curve is given by $(\sqrt{2} s / 2, \sqrt{2} s / 2)$ which corresponds to the minimal quadratic cone. This proves the theorem. 


\section{REFERENCES}

1. H. Alencar, Hipersuperficies minimas de $\mathbf{R}^{2 m}$ invariantes por $\mathrm{SO}(m) \times \mathrm{SO}(m)$, Tese de Doutorado, IMPA, 1988.

2. A. Back, M. do Carmo, and W. Y. Hsiang, On some fundamental equations in equivariant Riemannian geometry, Preprint.

3. J. L. Barbosa and M. do Carmo, Helicoids, catenoids and minimal hypersurfaces of $\mathbf{R}^{n}$ invariant by an l-parameter group of motions, An. Acad. Brasil. Ciênc. 53 (1981), 403-408.

4. E. Bombieri, E. De Giorgi, and E. Giusti, Minimal cones and the Bernstein problem, Invent. Math. 7 (1969), 243-268.

5. J. de M. Gomes, Sobre hipersuperficies com curvatura média constante no espaço hiperbólico, Tese de Doutorado, IMPA, 1984.

6. J. K. Hale, Ordinary differential equations, rev. ed., Krieger, 1980.

7. W. T. Hsiang and W. Y. Hsiang, On the existence of codimension one minimal spheres in compact symmetric spaces of rank 2, J. Differential Geom. 17 (1982), 583-594.

8. W.Y. Hsiang, Z. H. Teng, and W. C. Yu, New examples of constant mean curvature immersions of $(2 k-1)$-spheres into Euclidean $2 k$-space, Ann. of Math. (2) 117 (1983), 609-625.

9. J. Palis and W. de Melo, Geometric theory of dynamical systems, Springer-Verlag, 1982.

Departamento de Matemática, Universidade Federal de Alagoas, 57000 MaceióALAGOAS, Brasil

E-mail address: has@ima.ufal.alan.br

Instituto de Matemática Pura e Aplicada (IMPA), Estrada Dona Castorina, 110, 22460 Rio DE JANEIRo, BRASIL 